\title{
The probability for a Pap test to be abnormal is directly proportional to HPV viral load: results from a Swiss study comparing HPV testing and liquid-based cytology to detect cervical cancer precursors in 13842 women
}

\author{
G Bigras*,I and F de Marval ${ }^{2}$ \\ 'Laboratoire Cytopath, Unilabs SA, 12 Place Cornavin, Genève I, CH-I2II, Switzerland; '²aboratoire Bioanalytique-Riotton, Unilabs SA, 12 Place \\ Cornavin, Genève I, $\mathrm{CH}-121$ I, Switzerland
}

In a study involving 13842 women and I I 3 gynaecologists, liquid-based cytology and HPV testing for detecting cervical cancer were compared. A total of 1334 women were found to be positive for one or both tests and were invited for colposcopy with biopsy. A total of 1031 satisfactory biopsies on 1031 women were thereafter collected using a systematic biopsy protocol, which was random in the colposcopically normal-appearing cervix or directed in the abnormal one. In all, 502 women with negative tests were also biopsied. A total of 82 histologic high-grade squamous intraepithelial lesion (HSIL) were reported in biopsies, all from the group with one or both tests positive. Sensitivity and specificity to detect histologic HSIL were 59 and 97\% for cytology, and 97 and $92 \%$ for HPV. In total, I4\% of reviewed negative cytological preparations associated with histologic HSIL contained no morphologically abnormal cells despite a positive HPV test. This suggested a theoretical limit for cytology sensitivity. HPV viral load analysis of the I I43 HPVpositive samples showed a direct relationship between abnormal Pap test frequency and HPV viral load. Thus, not only does the HPV testing have a greater sensitivity than cytology but the probability of the latter being positive can also be defined as a function of the associated HPV viral load.

British Journal of Cancer (2005) 93, 575-581. doi:I0.1038/sj.bjc.6602728 www.bjcancer.com

Published online 2 August 2005

(c) 2005 Cancer Research UK

Keywords: HPV; liquid-based cytology; viral load sensitivity

The Pap test is currently central to the detection of cervical cancer precursors. Conventional smears, whose utilisation started in the early 1950s, have been progressively replaced by liquid-based methods over the past 10 years. Discovery of the role of HPV in the natural history of cervical cancer and the ability to detect it in liquid cytology medium are rapidly modifying the standard in cervical cancer detection. HPV detection is nowadays mainly used in atypical squamous cells of undetermined significance (ASC-US) triage. The next step will be the utilisation of combined HPV testing and cytology to detect cervical cancer and its precursors. After 50 years of cytology as the sole test in cervical cancer prevention, new methods are appearing to palliate the Achilles' heel of cytology, which is sensitivity. Indeed, many reports (Belinson et al, 1999; Kuhn et al, 2000; Ratnam et al, 2000; Schiffman et al, 2000; Wright et al, 2000; Clavel et al, 2001; Kulasingam et al, 2002; Petry et al, 2003) have established a higher sensitivity for HPV than for cytology, although published results are not unanimous (de Cremoux et al, 2003). The main study

* Correspondence: Dr G Bigras, Dynacare Kasper Medical Laboratories, \#200, 10150 - 102 Street, Edmonton, Alberta, Canada T5J 5E2;

E-mail: gilbert_bigras@yahoo.com

Received I2 May 2005; revised I July 2005; accepted I July 2005; published online 2 August 2005 objective was to confirm the better sensitivity of HPV testing, especially in the context of routine clinical practice.

Between April 2002, and January 2004, a prospective clinical study was conducted in order to compare two tests for the detection of cervical cancer precursors: a liquid-based cytology preparation, Surepath ${ }^{\circledR}$ (Tripath Imaging, Burlington, NC, USA) and a test for high-risk HPV DNA, Hybrid Capture ${ }^{\circledR}$ II (Digene Corporation, Gaithersburg, MD, USA). The investigation was conducted in the laboratory Cytopath based in Geneva, Switzerland, a private laboratory member of the Unilabs Group. Cytopath has used liquid-based cytology since 1996, analysing approximately 100000 slides per year.

\section{MATERIAL AND METHODS}

A target of 15000 cytologic samples was established in the hope of collecting approximately 250 histologic HSIL, providing adequate power to estimate the differences, if any, in performance characteristics between the tests. Women 30 or more years of age had been favoured for study participation in order to optimise HSIL collection. Most study participants had been screened yearly with liquid-based cytology starting at least 5 years before this study. They were thereby considered to be at low risk for cervical 
cancer. Recruitment was not from sexually transmitted disease clinics or other high-risk populations. Study participants were recruited by 113 gynaecologists from six Swiss cantons (Genève, Vaud, Neuchâtel, Fribourg, Valais and Tessin), most of whom are in private practice. Participants were given the option of study participation following a discussion with their gynaecologists of the risks and benefits of enrolment, and the completion of the informed consent document. Prior to initiation, the study was reviewed and approved by the Ethics Committee of the 'Association des Médecins du canton de Genève'.

\section{Liquid-based cytology}

All cervical samples were taken using the Surepath ${ }^{\circledR}$ (Tripath Imaging, Burlington, NC, USA) liquid-based preparation. In agreement with the manufacturer, the gynaecologists put the collecting device in the Surepath vial after having performed a cervical scrape. Cervex Brush ${ }^{\circledR}$ (CooperSurgical Inc., Trumbull, CT, USA) was the only provided collecting device. All vials were sent to the Cytopath Laboratory and prepared according to the protocol recommended by the manufacturer. A homogenised fraction of the sample was used to prepare the cytological preparation with the AutocytePrep ${ }^{\circledR}$ processor. The residual cellular material underwent centrifugation and was resuspended in $500 \mu \mathrm{l}$ distilled water for further HPV testing. In the text, 'CYTO' refers to the Surepath ${ }^{\circledR}$ liquid-based cytology test.

\section{HPV testing}

HPV testing was performed at the Bioanalytique-Riotton Laboratory (another member of the Unilabs group). Cell suspensions were stored at $2-8^{\circ} \mathrm{C}$ for up to 1 week before HPV testing. The HPV DNA assay was performed blind to cytologic and histologic diagnoses. Specimens were tested for high- and intermediate-risk HPV types $(16,18,31,33,35,39,45,51,52,56,58,59$ and 68$)$ using the Digene High-Risk HPV DNA Test Hybrid Capture ${ }^{\mathbb{R}}$ II (Digene Corporation, Gaithersburg, MD, USA). The DNA assay was processed according to the manufacturer's instructions, using microtubes and a water bath for hybridisation and manual washing. In agreement with the kit manufacturer, clinical specimens were denatured for $90 \mathrm{~min}$ in order to reduce borderline results. Relative light units (RLU) were measured on a DML 2000 luminometer (Digene Corporation, Gaithersburg, MD, USA). Quality control was assessed using the manufacturer's assay calibration criteria and by the regular use of HC2 HPV DNA Test Panel (Digene Corporation, Gaithersburg, MD, USA). The cutoff value was calculated as the mean RLU value of the calibrator $\left(1.0 \mathrm{pg} \mathrm{ml}^{-1} \mathrm{HPV} 16 \mathrm{DNA}\right)$ tested in triplicate. Specimen RLU values were then converted into a ratio to the cutoff value. Specimens with a ratio $<1.00$ were considered negative. Specimens with ratios between 1.00 and 5.00 were systematically retested in duplicate and the mean of the three values was calculated. If discrepant RLU values were observed in the two tests, samples were tested in duplicate again (maximum three thawing cycles). Specimens with a final ratio $\geqslant 1.00$ were considered positive $(\mathrm{HPV}+)$. In the text, HPV always refers to the Hybrid Capture $^{\mathbb{R}}$ II test. Therefore, an HPV - might refer to the absence of HPV virus or to an undetected type by the Hybrid Capture ${ }^{\mathbb{R}}$ II test or detection of the virus at an RLU ratio $<1.00$. All positive RLU values were collected and inserted into the database in order to compare the viral load expressed in RLU with the cytologic and biopsy results.

\section{Cytology}

All the cytologic slides were read in the Cytopath Laboratory by one of 13 cytotechnicians who knew which cases in the study but did not have access to the HPV result (nor did any of the medical staff) before the cytologic report was sent to the gynaecologist. The cytologic interpretation was done according to the Bethesda 2001 recommendations (Solomon et al, 2002). Any result $\geqslant$ ASC-US was considered as a positive cytologic result $(\mathrm{CYTO}+)$. If either test was positive, a request was sent for colposcopy with biopsy. To permit computation of true test sensitivity and specificity, disease prevalence among the women with both tests negative was assessed by performing colposcopy and biopsy on 502 randomly selected members of this group. At the end of the study, all cytologic preparations associated with a subsequent HSIL biopsy result were reviewed. The number of positive spots was retrospectively determined on each slide. A positive spot was defined as a single cell or a group of cells with sufficient atypia to be reported as at least an AS-CUS.

\section{Biopsy}

Before the beginning of the study, most of the 113 gynaecologists attended a formal review course on colposcopy procedure and interpretation taught by a Swiss expert colposcopist and educator. A biopsy was requested on all patients undergoing colposcopy: the biopsy would be directed if a lesion was noted (acetowhite epithelium, mosaicism, etc.), or random by strongly brushing the proximal endocervical canal if no lesion was visualised. In the latter case, the collecting device (a brush) was sent to the laboratory in formalin. At the laboratory, the brush was scraped with a scalpel and all material was paraffin blocked in order to obtain a histological preparation.

\section{Statistical methods}

Percentage values were described with $95 \%$ asymmetrical binomial confidence intervals computed with the Clopper-Pearson exact method. Student's $t$-test was used to compare mean values with a threshold of $5 \%$ for $P$-value to be significant. In order to correctly estimate the sensitivity and specificity of both tests, a method for correcting biopsy verification bias was used. Verification bias is caused by the fact that the percentages of biopsy verification vary considerably among HPV/CYTO subsets because of practical limitations. Indeed, only 4\% (502 out of 12508) of women HPV-/CYTO- and 77\% (1031 out of 1334) of women positive for at least one test, HPV +/CYTO-, HPV $-/ \mathrm{CYTO}+$ or $\mathrm{HPV}+1$ $\mathrm{CYTO}+$, underwent colposcopy with biopsy. Given the low prevalence of disease in this population, the bias causes a falsely decreased specificity for both tests. The correction relies on the estimation of lesion frequency found in each subset HPV/CYTO if all patients had been colposcoped and biopsied. The adjusted value (AHCL) was derived from the formula $A_{\mathrm{HCL}}=F_{\mathrm{HCL}}+U_{\mathrm{HC}}$ ${ }^{*}\left(F_{\mathrm{HCL}} /\left(F_{\mathrm{HC}(\mathrm{Normal})}+F_{\mathrm{HC}(\mathrm{LSIL})}+F_{\mathrm{HC}(\mathrm{HSIL})}\right)\right)$ (Ratnam et al, 2000). $\mathrm{L}$ is the biopsy result. FHCL is the frequency of lesion $\mathrm{L}$ obtained from the study without adjustment within a given HPV/CYTO subset. $U_{\mathrm{HC}}$ is the number of unverified patients in the same subset either because the biopsy was not received or because the biopsy was unsatisfactory.

\section{RESULTS}

\section{Cytology and HPV}

In total, 13865 liquid cytology specimens were received in the laboratory. Of these, 13842 were completed for both tests, CYTO and HPV. Overall, $9.6 \%$ of women (1334 out of 13842) were positive for one or both tests (CYTO or HPV), $8.2 \%$ of women (1143 out of 13842 ) were positive for HPV and $6.1 \%$ ( 841 out of 13842 ) were positive for HPV alone (HPV + CYTO-). In all, $96.4 \%$ (13345 out of 13842 ) of women were 30 years old or more, with a percentage of HPV infection of $7.9 \%$. This percentage rose to $17.4 \%$ in the 495 women younger than 30 years. For two women, 


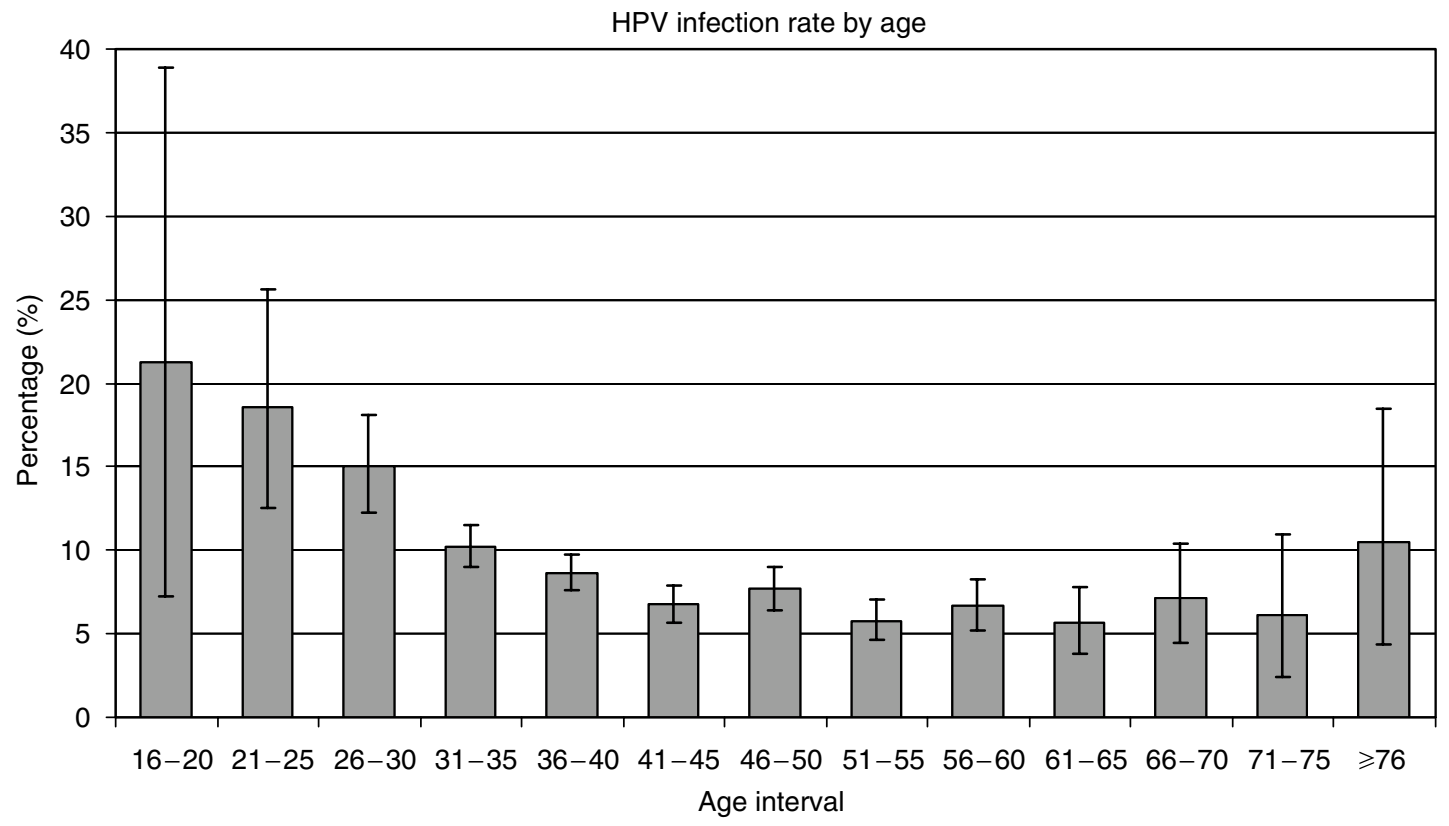

Figure I Percentage of HPV infection ( $y$-axis) against age intervals ( $x$-axis). 95\% confidence intervals are superposed to grey bars. The highest HPV infection rates are found in the youngest women. The percentage progressively declines as age increases $(n=13840)$.

the age was not available. Mean age of the 13840 women was 44.4 years (range 17-93). The higher frequency of HPV positivity in younger women is portrayed in Figure 1, where a progressive decline of HPV positivity is observed as age increases. All further results are unrelated to age. In total, $3.6 \%$ (493 out of 13842 ) of women were positive for CYTO and $1.4 \%$ (191 out of 13842 ) were positive for CYTO alone (HPV-CYTO +). Among the CYTO results, there were 243 ASC-US (1.8\%), 14 atypical squamous cells: cannot exclude HSIL (ASC-H) $(0.1 \%), 201$ low-grade squamous intraepithelial lesion (LSIL) (1.5\%), 30 high-grade squamous intraepithelial lesion (HSIL) $(0.2 \%)$ and five atypical glandular cells (AGC) $(0.04 \%)$.

\section{Biopsy results}

Of the 1334 women with abnormal tests, 1086 (81.4\%) underwent colposcopy with biopsy. Out of 1086, 1031 (95\%) biopsies were satisfactory for evaluation. A total of $18.5 \%$ women (248/ out of 1334) refused to be colposcoped, missed appointments or underwent the follow-up in other laboratories. In order to verify the negative group (HPV-CYTO-), random invitations for colposcopy were regularly sent to women with both tests negative during the study. Since some women declined to participate, up to 700 invitations were sent before being able to gather the target number of 500. Finally, 502 women with normal tests were colposcoped and biopsied. These biopsy results were divided into three categories: normal, LSIL (CIN 1) and HSIL (CIN2/3). No invasive lesion was found on cytology and biopsy in any group. The details of the results are presented in Table 1, where biopsy results are crossed to CYTO and HPV results. Table 2 presents biopsy results according to the HPV/CYTO subsets. A key result is that $41.5 \%$ of all histologic HSIL lesions were found in the subset HPV + CYTO-.

\section{Sensitivity, specificity and negative predictive value of CYTO and HPV}

The unbiased estimation of frequencies of lesions (normal, LSIL and HSIL) per HPV/CYTO subset for the entire study population (Table 3) permits calculation of unbiased sensitivity and specificity for detection of histologic HSIL (95\% confidence interval in parentheses). Sensitivity is $58.7 \%(48.6-68.2)$ for CYTO and $97 \%$ (91.8-99.4) for HPV, while specificity is $96.9 \%$ (96.6-97.2) for CYTO and 92.4\% (91.9-92.9) for HPV. The negative predictive value of CYTO and HPV are, respectively, 99.75 (99.67-99.83) and 99.98 (99.96-100). On the basis of the fact that no histologic HSIL lesion was found in the 502 randomly chosen women negative for both tests, the negative predictive value of the combination of negative tests HPV and CYTO estimated for all 13842 patients is $100 \%(99.98-100)$.

\section{Viral load expressed as RLU and logarithm RLU, compared with cytological and biopsy results}

The $1143 \mathrm{HPV}$-positive results have RLU values ranging from 1 to 3180. The distribution of RLU values is nonlinear. Most RLU values are small: cumulatively, $22 \%$ of all the HPV-positive results are less than $2.2 \mathrm{RLU}, 38 \%$ are less than $5.0 \mathrm{RLU}$ and $51 \%$ are less than 11.2 RLU. In order to compare the HPV distribution values more easily against frequency, the RLU values were transformed using the natural logarithm function. Therefore, the RLU interval $(1: 3200)$ becomes the $\operatorname{lnRLU}$ interval $(0: 8)$. The latter was subdivided in 10 subintervals as illustrated in Figure 2. In this figure, the black line with small white triangles represents the percentage of HPV + samples per lnRLU interval. This line shows that a majority of HPV-positive samples are associated with low HPV viral loads. The grey bars in Figure 2 represent the frequencies of positive CYTO against the HPV viral load expressed in lnRLU. A clearcut relationship is observed: the CYTO positivity frequency is directly related to the viral load. In the last subinterval [7.2-8.0], all the $28 \mathrm{HPV}+$ samples were always associated with a positive CYTO test ( $\geqslant$ ASC-US).

The black line with small white squares in Figure 2 represents the CYTO false negatives with subsequent biopsy proven HSIL. The $95 \%$ confidence intervals are not drawn (to avoid obscuring the chart) but are enumerated thereafter in square brackets. Per increasing lnRLU interval, the frequencies of histologic HSIL (CYTO negative) are $2 / 5=40 \%$ [12-77], 5/5 $=100 \%$ [56-100], $5 / 6=83 \% \quad[35-99], 8 / 10=80 \% \quad[44-97], 8 / 17=47 \% \quad[23-72]$, $6 / 11=54 \%[23-83], 0 / 9=0 \%[0-28], 0 / 5=0 \%[0-45], 0 / 6=0 \%$ 
Table I Biopsy results $(n=1533)$ including control group crossed to Pap results (based on Bethesda 200I) and HPV results

PAP cytology results

\begin{tabular}{|c|c|c|c|c|c|c|c|}
\hline Biopsy & Normal & ASC-US & ASC-H & LSIL & HSIL & AGC & Tota \\
\hline AIS & 0 & 0 & 0 & 0 & 0 & 0 & 0 \\
\hline CIN3 & 0 & I & 0 & 0 & 0 & 0 & I \\
\hline $\mathrm{CINI}$ & 11 & 39 & 1 & 7 & 0 & 0 & 58 \\
\hline Normal & 491 & 63 & 0 & 9 & 1 & 2 & 566 \\
\hline \multicolumn{8}{|c|}{$\mathrm{HPV}+(n=907)$} \\
\hline AlS* & 0 & 1 & 0 & 0 & 0 & 2 & 3 \\
\hline CIN3 & 25 & 6 & I & 7 & 16 & 0 & 55 \\
\hline CIN2 & 9 & 2 & 2 & 3 & 6 & 0 & 22 \\
\hline $\mathrm{CINI}$ & 240 & 30 & I & 83 & 3 & 0 & 357 \\
\hline Normal & 393 & 24 & I & 50 & 2 & 0 & 470 \\
\hline
\end{tabular}

ASC-US = atypical squamous cells of undetermined significance; ASC-H = atypical squamous cells: cannot exclude HSIL; LSIL = low-grade squamous intraepithelial lesion; $\mathrm{HSIL}=$ high-grade squamous intraepithelial lesion; AGC = atypical glandular cells. *The three AIS (in situ adenocarcinoma) were found combined with HSIL (CIN3) lesions within each biopsy.

Table 2 Biopsy results according to subsets HPV/CYTO

\begin{tabular}{|c|c|c|c|c|c|}
\hline Category results & Results (n) & Satisfactory biopsies $(n)$ & Normal biopsies & LSIL biopsies & HSIL biopsies \\
\hline HPV-CYTO+ & 191 & 124 & $75(\mid 15.5)$ & $47(72.4)$ & $2(3.1)$ \\
\hline Total & 13842 & 1533 & $1036(12941.8)$ & 415 (796.3) & $82(103.9)$ \\
\hline
\end{tabular}

Adjusted values $\left(A_{H C L}\right)$ for verification bias are in parentheses. They derived from the formula $A_{H C L}=F_{H C L}+U_{H C} *\left(F_{H C L} /\left(F_{H C(n o r m a l)}+F_{H C(L S I)}+F_{H C(H S I L)}\right)\right)$. $L$ is the biopsy result. $F_{H C L}$ is the frequency of lesion L obtained from the study without adjustment within a given HPV/CYTO subset. $U_{H C}$ is the number of patients in the same subset unverified

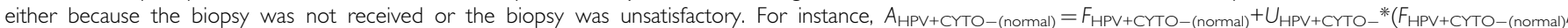
$\left(F_{\mathrm{HPV}+\mathrm{CYTO}-(\text { normal) }}+F_{\mathrm{HPV}+\mathrm{CYTO}-(\mathrm{LSLL})}+F_{\mathrm{HPV}+\mathrm{CYTO}-(\mathrm{HSL}))}\right)=393+(84 \mathrm{I}-667) *(393 /(393+240+34))=495.5$ (see text in statistical method for verification bias explanation). $\mathrm{HSIL}=$ high-grade squamous intraepithelial lesion; LSIL = low-grade squamous intraepithelial lesion.

Table 3 Frequencies of true positive, true negative, false positive and false negative for HPV and CYTO tests (for HSIL detection) where HPV and CYTO represent adjusted values ( $A_{H C L}$, see Table 2 for biopsy verification bias)

Test frequencies to detect CIN2/3 (HSIL) with related sensitivity and specificity adjusted with verification bias

\begin{tabular}{lrrlr}
\hline Result & \multicolumn{1}{c}{ HPV } & \multicolumn{1}{c}{ CYTO } & \multicolumn{2}{c}{$\begin{array}{c}\text { Sensitivity and specificity to } \\
\text { detect HSIL }\end{array}$} \\
\hline True positive & 100.8 & 61.0 & Sensitivity CYTO & $58.7 \%$ \\
True negative & 12695.9 & 13306.1 & Specificity CYTO & $96.9 \%$ \\
False positive & 1042.2 & 432.0 & Sensitivity HPV & $97.0 \%$ \\
False negative & 3.1 & 42.9 & Specificity HPV & $92.4 \%$ \\
\hline
\end{tabular}

95\% confidence interval: sensitivity HPV $=[91.8-99.4 \%]$, sensitivity CYTO $=[48.6-$ $68.2 \%]$, specificity HPV $=[91.9-92.9 \%]$ and specificity CYTO $=[96.6-97.2 \%]$.

$[0-39]$ and $0 / 5=0 \%[0-45]$. For $\operatorname{lnRLU}$ values $\geqslant 4.8,25$ histologic HSIL were found and all were detected by CYTO.

The black line with small white circles in Figure 2 represents the CYTO false negatives with subsequent biopsy proven LSIL. The 95\% confidence intervals are not drawn as previously, but are enumerated thereafter in square brackets. Per increasing lnRLU interval, the frequencies of histologic LSIL (CYTO negative) are $67 / 79=84 \%[75-92], 44 / 57=77 \%$ [64-87], 34/42 $=80 \%$ [66-91], $38 / 47=80 \%$ [67-91], $16 / 30=53 \%$ [34-72], $16 / 28=57 \%[37-76]$,
$9 / 19=47 \% \quad[24-71], 9 / 20=45 \% \quad[23-68], 7 / 26=27 \% \quad[12-48]$ and $0 / 11=0 \%[0-24]$.

\section{Retrospective analysis of cytological preparations associated with HSIL}

Figure 3 portrays the retrospective analysis of the 79 cytological preparations (CYTO-HPV + and $\mathrm{CYTO}+\mathrm{HPV}+$ ) associated with biopsy-proven HSIL (one cytological preparation, $\mathrm{HPV}+\mathrm{CYTO}+$, was not available for spot counting). The mean number of positive spots was 5.2 for $\mathrm{HPV}+/ \mathrm{CYTO}-$ cytological preparations (black triangles, $n=34$ ) and 23.1 for $\mathrm{HPV}+1$ CYTO + cytological preparations (white squares, $n=45$ ). A total of $14 \%$ (11 out of 79 ) of cytological preparations HPV + /CYTOdid not show any positive spots. The mean $\operatorname{lnRLU}$ values were 2.6 for cytological preparations $\mathrm{HPV}+/ \mathrm{CYTO}-$ (black triangles, $n=34$ ) and 4.8 for $\mathrm{HPV}+/ \mathrm{CYTO}+$ cytological preparations (white squares, $n=45$ ). Both differences (number of positive spots and $\operatorname{lnRLU}$ ) were found significant with $t$-test $(P<0.05)$. The two cytological preparations $\mathrm{HPV}-/ \mathrm{CYTO}+$ associated to biopsyproven HSIL had six and 10 positive spots (not shown in Figure 3).

\section{DISCUSSION}

It is important to note that the methodology of the present study required that the collecting device be kept in the vial so that $100 \%$ 


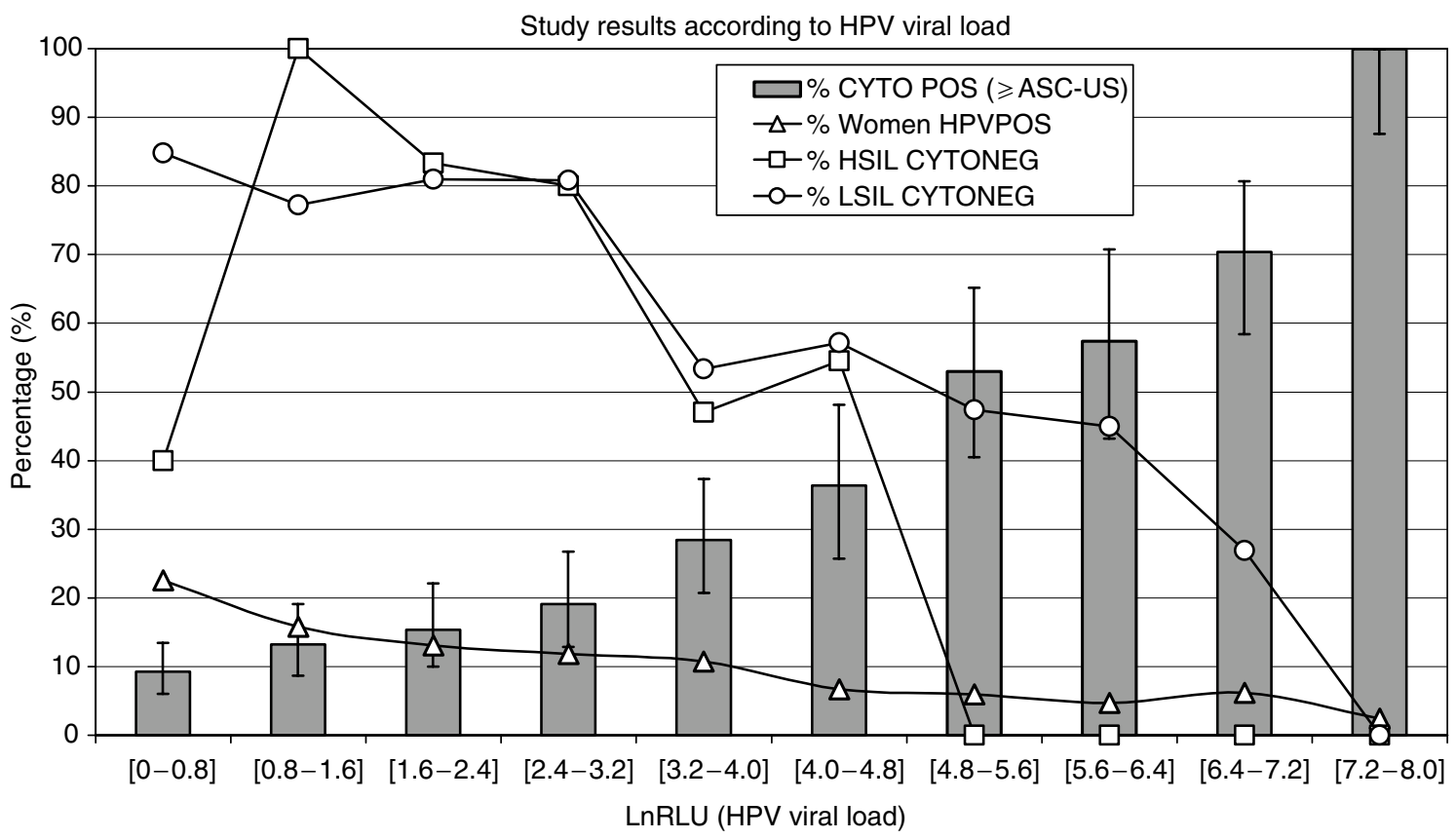

Figure 2 Percentage (y-axis) against HPV viral load of II43 HPV + samples (x-axis) reported as Logarithm value of Relative Light Unit (LnRLU). The '\% women HPVPOS' curve shows the distribution of the HPV + samples among LnRLU intervals. In this study, most HPV + samples have low LnRLU: near $50 \%$ of HPV + samples are found in the first three LnRLU intervals. The grey bars represent the percentage per LnRLU interval of positive cytological preparation ( $\geqslant$ ASC-US); it shows a direct relationship between the HPV viral load and the probability for a Pap test to be positive. The HSIL CYTONEG and LSIL CYTONEG curves represent the percentage per LnRLU interval of false negative cytological preparations with subsequent biopsy proven lesion.

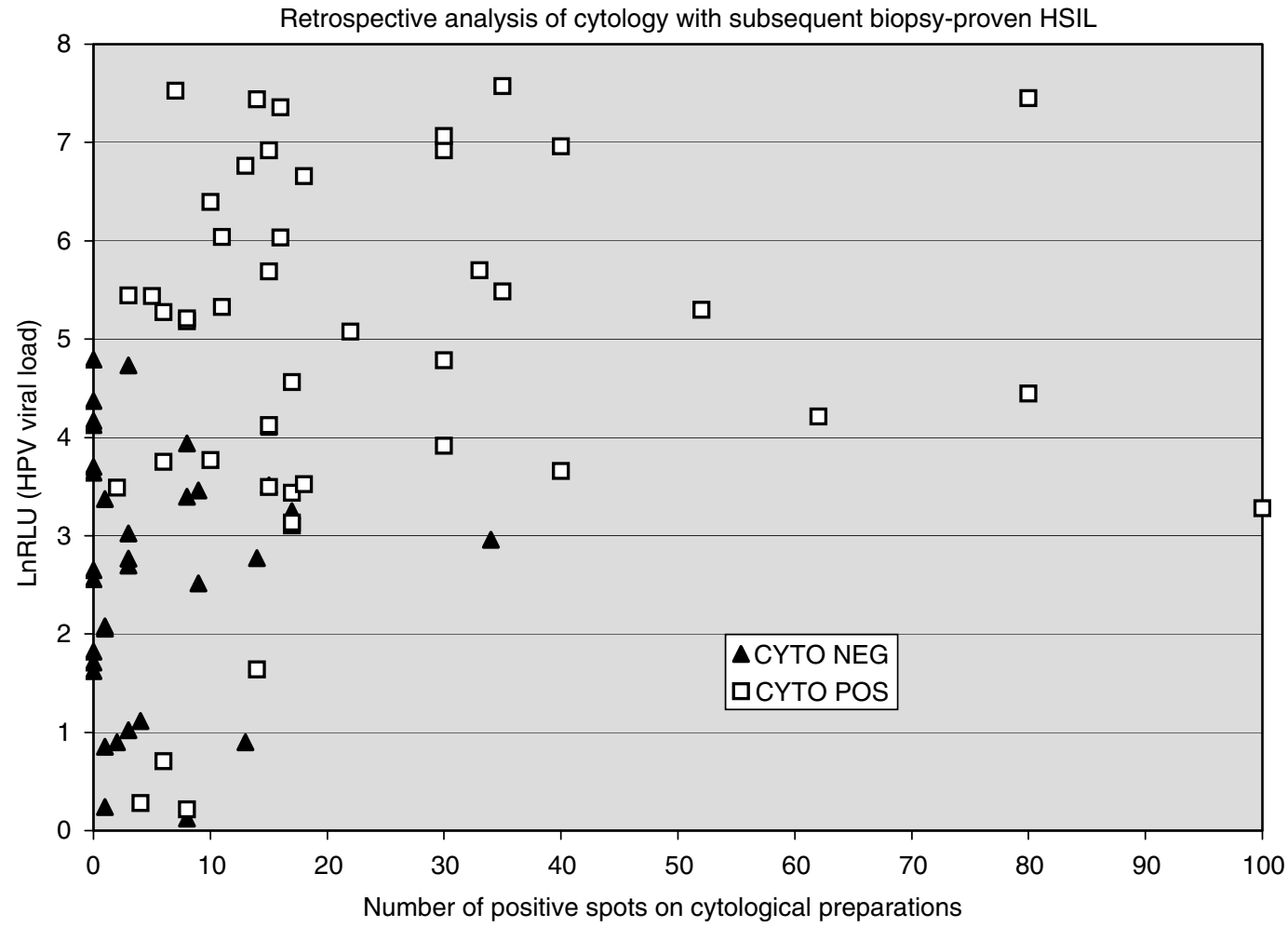

Figure 3 Retrospective analysis of all cytological preparations associated with subsequent histological HSIL. Logarithm values of RLU (y-axis) against number of positive cellular spots ( $x$-axis) retrospectively counted on cytological preparations. False negative CYTO (black triangles) have a smaller viral load and fewer number of positive cellular spots than true positive CYTO (white squares). Both differences are statistically significant. However, a significant number of false negative cytological preparations (I I out of $34(32 \%)$ ) have no cellular spots despite a significant viral load $(n=79)$. 
of the cervical scrape was sent to the laboratory. The conventional smear, by selecting a random fraction of the cervical scrape, involves an important loss of material (Goodman and Hutchinson, 1996; Al-Awadhi et al, 2001). LBC technique may also reproduce the flaws of the conventional smear. It was demonstrated that when discarding the collecting device, a mean of $37 \%$ of the cervical scrape material is lost. Worse, often the lost material comes preferentially from the transformation zone (Bigras et al, 2003).

The relationship between the percentage of HPV infection and the age shown in Figure 1 is similar to those found in other studies except for the oldest group. This last interval is based on a reduced number of women as shown by its relatively wide confidence interval. Furthermore, it is hypothesised that this high frequency is probably biased because these older women are likely to be those who had previous abnormal tests and probably form a nonrandom sample of this age group.

\section{Contribution of HPV viral load study}

At the sample level, the study of HPV viral load explains the reason that CYTO has inferior sensitivity. As shown in Figure 2, the probability of detecting abnormal cell(s) in an LBC preparation is a function of the associated HPV viral load. These results are concordant with the work of Schlecht et al (2003). As HPV is an intracellular virus, the viral load is proportional to the number of infected cells. Then, the higher the viral load, the greater the number of infected cells on the slide and the greater the probability for the LBC test to be positive. However, the viral load per cell varies between the LSIL and HSIL cells. LSIL is associated with active viral genome amplification with up to 1000 viral copies per keratinocyte (Garner-Hamrick and Fisher, 2002), while HSIL is associated with host DNA integration with few viral DNA per keratinocyte (Jeon et al, 1995). Then, an HSIL-associated sample would contain many more abnormal cells than an LSIL-associated sample with identical HPV viral load. This would explain why from 4.8 lnRLU value and higher (Figure 2), there are no more histologic HSIL false negative CYTO samples but still many LSIL false negative CYTO samples.

While some false negative cytological preparations can be explained by human misreading, most are not. False negative preparations would contain few abnormal cells and relatively low HPV viral load. Inspection of Figure 3 partly supports this hypothesis. First, it is reasonable, although arbitrary, to accept that missing up to three abnormal cells among 30000-50000 cells is independent of cytotechnician reading performance. Therefore, $64 \%$ (22 out of 34 ) of false negative CYTO with subsequent histologic HSIL were independent of cytotechnician reading performance. Furthermore, up to $14 \%$ (11 out of 79) of cytological preparations showed no abnormal cells despite relatively high viral loads. It is then hypothesised that infected cells are present on the slides but do not always show sufficient morphologic anomalies to be recognised. This concept is supported in the literature (Ward et al, 1990; Cramer et al, 1997; Salvia et al, 2004).

At the population level, the study of HPV viral load explains why cytology sensitivity varies considerably among reports. The relationship between the probability that a Pap test is abnormal and the viral load is thought to be independent from intrinsic factor related to this study. Conversely, the viral load distribution (Figure 2: black line with white triangles) is unique to the studied population. Its left-shifted pattern (indicating that a majority of $\mathrm{HPV}+$ samples have small viral load) would be the hallmark of a low-risk population. If the same study was repeated with a highrisk population, it can be expected that the viral load distribution would be shifted to the right. Then, the number of abnormal cells per sample would be much more important and, consequently, the sensitivity gap between HPV and cytology for detection of HSIL would be much smaller. This idea is supported in the literature, as studies performed in a high-risk population demonstrated relatively small gaps (4,10, 10 and 15\%) (Belinson et al, 1999; Kuhn et al, 2000; Schiffman et al, 2000; Wright et al, 2000) compared with studies performed in a low-risk population with higher gaps (30, 38, 54 and 55\%) (Ratnam et al, 2000; Clavel et al, 2001; Kulasingam et al, 2002; Petry et al, 2003).

\section{HPV/CYTO subsets}

Derived from Table 2, the subsets HPV $+/ \mathrm{CYTO}+$ and HPV $+I$ CYTO- represent, respectively, 2.18 and $6.07 \%$ of the studied population. HSIL form $19.2 \%$ of biopsies in the HPV + /CYTO + subset and $5.1 \%$ in the HPV $+/ \mathrm{CYTO}$ - subset. Combining these results, one computes a ratio women/HSIL of 5.2 for $\mathrm{HPV}+1$ $\mathrm{CYTO}+$ and 19.6 for HPV + /CYTO - subsets. The large number of women without HSIL in the HPV + /CYTO- subset raises a difficulty in the clinical management. Wright et al (2004) have suggested an interim guidance for this issue. They suggested that colposcopy should not be performed in the setting routinely. HPV, along with cytology, should be repeated at 6-12 months. If either test is still positive, then colposcopy should be performed. Finding rare HSIL in the HPV-/CYTO + subset illustrates that rare HPV subtypes not detected by HC2 may cause HSIL. Cytology must then still be part of screening strategy. No HSIL was found in 502 biopsies from the HPV-/CYTO- subset. This is concordant with the Guanacaste study (Bratti et al, 2004: 0 HSIL out of 150) and the Shanxi study (Belinson et al, 1999 (Dr R Gerald Pretorius, personal communication, January 2005): 0 HSIL out of 1274). In total, no HSIL was found in 1926 biopsies from HPV-/CYTO- subset. The 95\% confidence interval, based on these 1926 biopsies, for the negative predictive value of the two tests together for histologic HSIL, is therefore $99.84-100.00 \%$.

\section{CONCLUSIONS}

This study found a higher sensitivity for HPV than liquid-based cytology testing in the detection of cervical cancer precursors. This adds to previous similar reports with the distinction that our study involved a nonacademic setting, thereby demonstrating that the method is robust and readily applicable as a routine test. By collecting all RLU values, a clearcut correlation between the probability for a Pap test to be positive and the associated HPV viral load was found. The findings can also explain how cytology sensitivity can vary among different studies since different populations will differ in their viral load distributions: at one extreme, cytology performs badly when the screened population is at low - risk, while at the other extreme, cytology performance would be much closer to that of HPV testing in a high-risk population. Rare HSIL are not detected by HC2, these cases being presumably related to HPV types that are not detected by the cocktail of probes used in HC2. The combination of Pap and HPV testing identifies all of the women harbouring HSIL that can be detected by the best colposcopic methods currently available, and thereby permits investigators and clinicians to focus their efforts on the population at risk, while reassuring the majority of women who are negative on both tests.

\section{ACKNOWLEDGEMENTS}

We thank first and foremost the women and the gynaecologists who participated in this study. We acknowledge the vital logistical support provided by the Cytopath and Bioanalytique-Riotton Laboratories staff. We thank all of the medical representatives who fostered this study in different Swiss cantons. We also thank the headquarters staff of Unilabs, and the Digene Corporation, Gaithersburg, MD, USA, who strongly supported the study. 


\section{REFERENCES}

Al-Awadhi R, Byrne M, Coleman DV (2001) The preparation of additional smears from a cervical scrape: impact on the rate of detection of cervical neoplasia. Cytopathology 12: $151-156$

Belinson J, Qiao Y, Pretorius R, Zhang W, Keaton K, Elson P, Fischer C, Lorincz A, Zahniser D, Wilbur D, Pan Q, Li L, Biscotti C, Dawson A, Li A, Wu L, Ling Y, Ma CP, Yang XP (1999) Prevalence of cervical cancer and feasibility of screening in rural China: a pilot study for the Shanxi Province Cervical Cancer Screening Study. Int J Gynecol Cancer 9(5): $411-417$

Bigras G, Malgorzata AR, Lambercy JM, Kunz B, Chatelain JP, Reymond O, Cornaz D (2003) Keeping collecting device in liquid medium is mandatory to ensure optimized liquid-based cervical cytologic sampling. J Lower Genital Tract Dis 7(3): 168-174

Bratti MC, Rodriguez AC, Schiffman M, Hildesheim A, Morales J, Alfaro M, Guillen D, Hutchinson M, Sherman ME, Eklund C, Schussler J, Buckland J, Morera LA, Cardenas F, Barrantes M, Perez E, Cox TJ, Burk RD, Herrero R (2004) Description of a seven-year prospective study of human papillomavirus infection and cervical neoplasia among 10000 women in Guanacaste, Costa Rica. Rev Panam Salud Publica 15(2): 75 - 89

Clavel C, Masure M, Bory JP, Putaud I, Mangeonjean C, Lorenzato M, Nazeyrollas P, Gabriel R, Quereux C, Birembaut P (2001) Human papillomavirus testing in primary screening for the detection of high-grade cervical lesions: a study of 7932 women. Br J Cancer 84(12): 1616-1623

Cramer HM, Skinner-Wannemuehler SE, Brown DR, Katz BP, Fife KH (1997) Cytomorphologic correlates of human papillomavirus infection in the 'normal' cervicovaginal smear. Acta Cytol 41(2): 261-268

de Cremoux P, Coste J, Sastre-Garau X, Thioux M, Bouillac C, Labbe S, Cartier I, Ziol M, Dosda A, Le Gales C, Molinie V, Vacher-Lavenu MC, Cochand-Priollet B, Vielh P, Magdelenat H (2003) Efficiency of the hybrid capture 2 HPV DNA test in cervical cancer screening. A study by the French Society of Clinical Cytology. Am J Clin Pathol 120(4): $492-499$

Garner-Hamrick PA, Fisher C (2002) HPV episomal copy number closely correlates with cell size in keratinocyte monolayer cultures. Virology 301: $334-341$

Goodman A, Hutchinson ML (1996) Cell surplus on sampling devices after routine cervical cytologic smears. A study of residual cell populations. I Reprod Med 41: 239-241

Jeon S, Allen-Hoffman BL, Lambert PF (1995) Integration of human papillomavirus type 16 into the human genoma correlates with a selective growth advantage of cells. J Virol 69: 2989-2997
Kuhn L, Denny L, Pollack A, Lorincz A, Richart RM, Wright TC (2000) Human papillomavirus DNA testing for cervical cancer screening in lowresource settings. J Natl Cancer Inst 92(10): 818-825

Kulasingam SL, Hughes JP, Kiviat NB, Mao C, Weiss NS, Kuypers JM, Koutsky LA (2002) Evaluation of human papillomavirus testing in primary screening for cervical abnormalities: comparison of sensitivity, specificity, and frequency of referral. JAMA 288(14): 1749-1757

Petry KU, Menton S, Menton M, van Loenen-Frosch F, de Carvalho Gomes H, Holz B, Schopp B, Garbrecht-Buettner S, Davies P, Boehmer G, van den Akker E, Iftner T (2003) Inclusion of HPV testing in routine cervical cancer screening for women above 29 years in Germany: results for 8466 patients. Br J Cancer 88(10): $1570-1577$

Ratnam S, Franco EL, Ferenczy A (2000) Human papillomavirus testing for primary screening of cervical cancer precursors. Cancer Epidemiol Biomarkers Prev 9(9): 945 -951

Salvia PN, Bergo SM, Bonesso-Sabadini PI, Tagliarini EB, Hackel C, De Angelo Andrade LA (2004) Correlation between histological criteria and human papillomavirus presence based on PCR assay in cervical biopsies. Int J Gynecol Cancer 14(1): 126-132

Schiffman M, Herrero R, Hildesheim A, Sherman ME, Bratti M, Wacholder S, Alfaro M, Hutchinson M, Morales J, Greenberg MD, Lorincz AT (2000) HPV DNA testing in cervical cancer screening: results from women in a high-risk province of Costa Rica. JAMA 283(1): 87-93

Schlecht NF, Trevisan A, Duarte-Franco E, Rohan TE, Ferenczy A, Villa LL, Franco EL (2003) Viral load as a predictor of the risk of cervical intraepithelial neoplasia. Int J Cancer 103(4): 519-524

Solomon D, Davey D, Kurman R, Moriarty A, O'Connor D, Prey M, Raab S, Sherman M, Wilbur D, Wright Jr T, Young N (2002) The 2001 Bethesda System: terminology for reporting results of cervical cytology. JAMA 287(16): $2114-2119$

Ward BE, Burkett B, Petersen C, Nuckols ML, Brennan C, Birch LM, Crum CP (1990) Cytologic correlates of cervical papillomavirus infection. Int J Gynecol Pathol 9(4): $297-305$

Wright TC, Denny L, Kuhn L, Pollack A, Lorincz A (2000) HPV DNA testing of self-collected vaginal samples compared with cytologic screening to detect cervical cancer. JAMA 283: $81-86$

Wright Jr TC, Schiffman M, Solomon D, Cox JT, Garcia F, Goldie S, Hatch K, Noller KL, Roach N, Runowicz C, Saslow D (2004) Interim guidance for the use of human papillomavirus DNA testing as an adjunct to cervical cytology for screening. Obstet Gynecol 103(2): 304-309 